\title{
Catalytic Resonance of Ammonia Synthesis by Dynamic Ruthenium Crystal Strain
}

\author{
Gerhard R. Wittreich*, Shizhong Liu*, \\ Paul J. Dauenhauer, ${ }^{\dagger,}{ }^{*}$, Dionisios G. Vlachos $\$, \beta, \xi^{*}$
}

Department of Chemical and Biomolecular Engineering, University of Delaware, 150 Academy St., Newark, DE 19716, USA

${ }^{\dagger}$ Department of Chemical Engineering and Materials Science, University of Minnesota, 421 Washington Ave SE, Minneapolis, Minnesota 55455, USA

${ }^{\beta}$ Catalysis Center for Energy Innovation, University of Delaware, 221 Academy Street, Newark, Delaware 19711, USA

${ }^{\xi}$ RAPID Manufacturing Institute and Delaware Energy Institute (DEI), University of Delaware, 221 Academy Street, Newark, DE 19711, USA

*Corresponding authors: hauer@umn.edu, vlachos@udel.edu

\begin{abstract}
Ammonia affords dense storage for renewable energy as a fungible liquid fuel provided it can be efficiently synthesized from hydrogen and nitrogen. In this work, the catalysis of ammonia synthesis was computationally explored beyond the Sabatier limit by dynamically straining a ruthenium crystal $( \pm 4 \%)$ at the resonant frequencies $\left(10^{2}\right.$ to $\left.10^{5+} \mathrm{Hz}\right)$ of $\mathrm{N}_{2}$ surface dissociation and hydrogenation. Density functional theory calculations at different strain conditions indicated that the energies of $\mathrm{NH}_{\mathrm{x}}$ surface intermediates and transition states scale linearly with that of the surface nitrogen on terraces, allowing the description of ammonia synthesis at a continuum of strain conditions. A microkinetic model including multiple sites and surface diffusion between step and $\mathrm{Ru}(0001)$ terrace sites of varying ratios for nanoparticles of differing size revealed that dynamic strain yields catalytic ammonia synthesis conversion and turnover frequency comparable to industrial reactors $\left(400{ }^{\circ} \mathrm{C}, 200 \mathrm{~atm}\right)$ but at lower temperature $\left(320^{\circ} \mathrm{C}\right)$ and an order of magnitude lower pressure $(20 \mathrm{~atm})$.
\end{abstract}

1.0 Introduction. The emergence of affordable electrical power from distributed wind turbines and solar panels requires a complementary chemical energy storage technology.(1) Electrochemical conversion of water to hydrogen affords a carbon-free energy storage medium, which can then be converted to liquid ammonia as an energy-dense, fungible liquid fuel.(2) Currently manufactured using hydrogen from methane via steam reforming, ammonia becomes more sustainable when associated carbon emissions are captured (blue ammonia) or when hydrogen is generated via carbon-free methods, such as water electrolysis (green ammonia).(3) These approaches to energy storage are best suited to small-scale modular microprocesses that can be located close to distributed renewable energy sources, providing immediate energy storage with the capability for energy-dense liquid transportation. $(4,5)$ But scaling down conventional ammonia synthesis is not economically viable primarily due to the poor performance of ammonia synthesis catalysts.( $($ )

Improving ammonia synthesis catalysts for new small-scale distributed applications requires improving the rate of reaction and single-pass reactor conversion. As a sequence of surface reactions including $\mathrm{N}_{2}$ dissociation and sequential hydrogenation, ammonia synthesis exhibits a Sabatier peak (Figure 1A) in catalytic turnover frequency consistent with a transition in ratelimiting surface reactions with ruthenium and iron near the peak. $(7,8)$ The resulting rate limitations on either side of the Sabatier peak then derive from the reduced rate of $\mathrm{N}_{2}$ dissociation and the hydrogenation of nitrogen-containing surface species.(9-11) Catalytic turnover frequency closer to the Sabatier peak has been obtained by tuning the catalyst surface energy using surface promoters or bimetallic catalysts such as CoMo.(12) However, the limitations of the conventional Haber-Bosch 

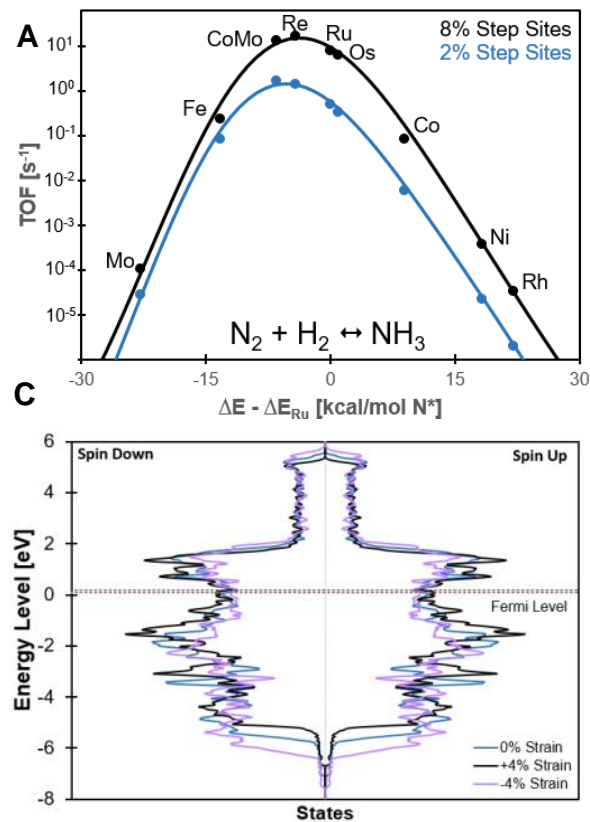
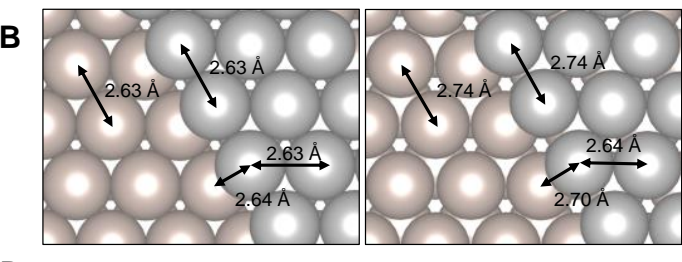

D

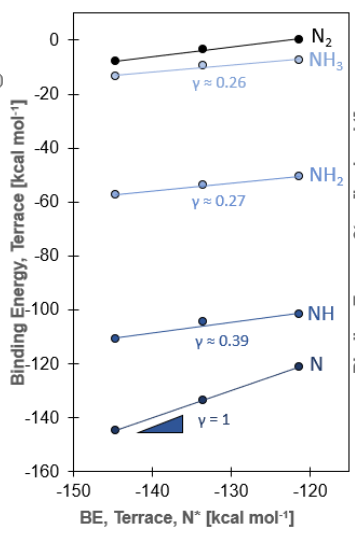

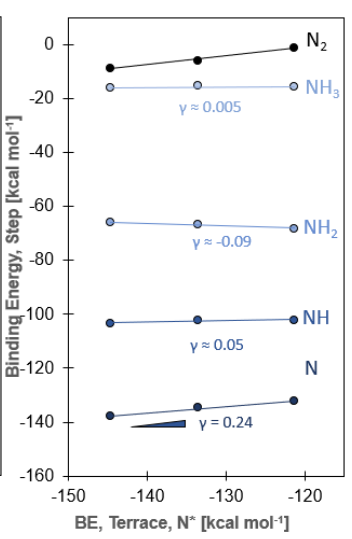

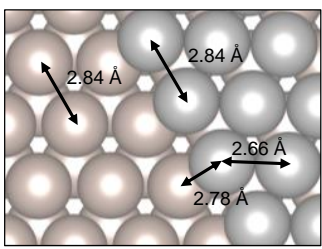

$\mathrm{E}$

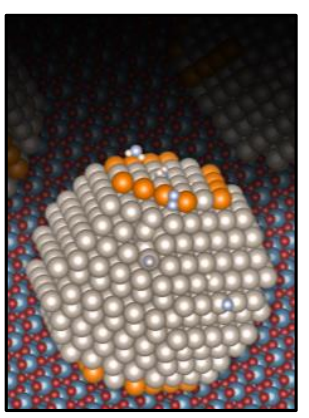

Figure 1. Catalytic synthesis of ammonia via strain. A. Sabatier volcano catalytic turnover frequency (TOF) at $0.31 \%$ conversion of $\mathrm{N}_{2}$ on supported metal nanoparticles with $2 \%$ or $8 \%$ step sites at 50 atm and $400{ }^{\circ} \mathrm{C}$; full details in the supporting information. B. Two-dimensional strain changes the atom-to-atom spacing $(-4,0,+4 \%$ spacing from left to right) across surface planes and steps. C. Two-dimensional strain shifts the d-band center of Ru by $\sim 0.23$ $\mathrm{eV}$. D. The binding energy of $\mathrm{NH}_{\mathrm{x}}(\mathrm{T})$ on $\mathrm{Ru}$ terrace sites varies slightly and possesses a low gamma $(\gamma<1)$ relative to $\mathrm{N}(\mathrm{T})$ on a clean terrace (low surface coverage). The binding energy $\mathrm{NHx}(\mathrm{S})$ on Ru step sites varies less than terrace sites due to the anisotropic behavior of step sites. E. Schematic of a typical Ru nanoparticle on alumina, designed via Wulff construction, showing step sites (orange) and various $\mathrm{NH}_{\mathrm{x}}$ adsorbates. These nanoparticles typically have 2$8 \%$ step sites (remainder terrace sites) for particle sizes of 8.0 to $2.5 \mathrm{~nm}$. The Wulff construction provides only an estimate of step sites for microkinetic calculations and is not used otherwise.

catalytic ammonia synthesis have led to alternative ammonia synthesis technologies including electrocatalysis(13) and plasma catalysis.(14)

One strategy to overcome the Sabatier limit is by crystal engineering whereby one purposely creates defects on the surface to create bifunctional catalysis(15-17) or preferred sites.(18) Another strategy for catalysts to achieve turnover frequencies beyond the Sabatier limit aims to oscillate the electronic state of the catalyst at frequencies conducive to the kinetics of surface chemistry. As recently described via simulation, $(19,20)$ oscillation of the catalyst electronic state between sides of the Sabatier peak can accelerate the overall reaction rate, control the extent of reaction, and even select specific reaction pathways in a reaction network. $(21,22)$ These dynamic catalysts exhibit a band of resonant applied frequencies yielding the highest catalytic turnover frequency unique to each catalytic reaction. Dynamic catalytic rate acceleration has already been demonstrated with dynamic formic acid electro-oxidation(23) and dynamic photocatalysis for methanol decomposition.(24)

Another method to dynamically alter the electronic state of catalysts is physical strain. Tensile or compressive strain yields physical and electronic rearrangement, described by the d-band center, $(25,26)$ leading to variable adsorbate binding energy. $(27,28)$ Evaluated computationally, the binding energy of common adsorbates, such as $\mathrm{CO}$ or $\mathrm{O}_{2}$, can vary as much as half of an electronvolt for large surface strain of 3-5\%,(29-31) with unique variation in binding energy for combinations of adsorbate, surface composition, and site structure.

Achieving strains sufficiently large to affect binding energy variation requires both a perturbation method and a strategy to prevent catalyst surface rearrangement under strain. Static strain can be implemented by lattice mismatch of overlayers with catalyst supports,(32-34) including the formation of subsurface alloys or core-shell particles. $(35,36)$ Dynamic strain can potentially be 
implemented by supporting a thin catalyst layer on temporally stressed supports. For example, deposition of a $\mathrm{Pt}$ overlayer on $\mathrm{LiCoO}_{2}$ exhibits a strain of $\sim 3 \%$ as lithium ions intercalate into and out of the cobalt oxide layers with applied potential.(37) Another variation is deposition on a physically deformed support, such as tungsten carbide achieving $>3 \%$ strain on a bendable poly(methyl methacrylate) diaphragm.(38) Strain is also dynamically applied via propagating surface acoustic waves, producing immense compressive and tensile strain regions when propagating through metal films.(39-42)

The challenge with implementing plastic deformation of catalytic materials emerges at high static strain, where rearrangement via dislocation motion can relax materials obviating the catalytic benefit of strained surfaces. However, in an oscillating stress field at sufficiently high frequency $\left(\sim 10^{-6} \mathrm{~s}\right)$, the thermal fluctuations of atoms required for dislocation motion are statistically improbable, thereby allowing for significantly higher dynamic stress and strain (>5\%) at short time intervals (e.g., millisecond-to-microsecond).(43, 44) Large strain at high frequencies (kilohertz to megahertz) is consistent with the requirements of resonant catalysis over this range of applied frequencies.

In this work, the ammonia synthesis reaction is computationally evaluated on ruthenium $(\mathrm{Ru})$ undergoing significant dynamic strain $( \pm 4 \%)$ to understand the impact on the catalytic turnover frequency and extent of reaction relative to equilibrium. The energies of surface intermediates and transition states were calculated under different magnitudes of compressive and tensile surface strain on both step sites (required for $\mathrm{N}_{2}$ dissociation $(45,46))$ and $\mathrm{Ru}(0001)$ terrace sites using density functional theory (DFT). The microkinetic model was then evaluated under dynamic strain with variable frequency and reactor temperature $\left(320-400{ }^{\circ} \mathrm{C}\right)$ and stoichiometric feed gas pressure (20-200 atm) while accounting for the effect of surface coverage and surface diffusion. Simulations indicate that dynamic ammonia synthesis at $20 \mathrm{kHz}$ and $320^{\circ} \mathrm{C}$ can match industrial reactor performance at an order of magnitude lower pressure (20 atm).

\subsection{Materials and Methods.}

2.1 Electronic Structure Calculations. Density functional theory (DFT) calculations were performed using the Vienna ab-initio Simulation Package (VASP)(47-49) code with the projector augmented wave method. The generalized gradient approximation (GGA) with the Perdew-BurkeErnzerhof (PBE)(50) functional was applied to describe exchange-correlation effects. The cutoff energy was $520 \mathrm{eV}$ and the convergence of energy and forces for geometry optimizations were set to $1 \times 10^{-5} \mathrm{eV}$ and $0.01 \mathrm{eV} / \AA$, respectively. For transition states searches, we used the climbing image nudged elastic band method (NEB) in VTST(51). The convergence criteria of energy and forces for NEB were set to $1 \times 10^{-5} \mathrm{eV}$ and 0.05 $\mathrm{eV} / \AA$, respectively. The transition states were confirmed using vibrational frequencies analysis.

A four-layer $\mathrm{Ru}(0001)$ slab with a $(3 \times 3)$ unit cell and a four-layer $\mathrm{Ru}(0001)$ slab with a $(3 \times 6)$ unit cell were employed, on which three of the six rows of top $\mathrm{Ru}$ atoms were removed to simulate the step site. A $3 \times 3 \times 1$ Monkhorst-Pack(52) was used for k-point sampling for the $(3 \times 3)$ unit cells and the $\mathrm{k}$ point was set to $2 \times 4 \times 1$ for $(3 \times 6)$ unit cells. All unit cells include a vacuum of $20 \AA$ between slabs. The surface strain was studied by changing the lattice constant of $\mathrm{Ru}$ slabs. Starting with the unstrained lattice constant of Ru bulk, compressive and expansive strain up to $4 \%$ were applied in the two principal directions parallel to the surface. For each model, the bottom two layers of $\mathrm{Ru}$ atoms were fixed, while the top two layers of $\mathrm{Ru}$ atoms with adsorbates were allowed to relax fully.

Electronic energies and vibrational data were computed for all surface species on terrace and step sites, and all transition states were located using NEB. Surface diffusions between step and terrace sites required multiple computations on adjacent binding sites away from the steps until terrace-like sites were reached. The diffusion step with the highest reaction barrier was used to model it as a single step.

2.2 Reaction Mechanism and Microkinetic Model. Microkinetic models (MKMs) were constructed for $0 \%$ (unstrained), $+4 \%$, and $-4 \%$ strain, including both $\mathrm{Ru}$ terrace and step sites. Scaling relationships were developed to model a continuum of strain levels. The MKM includes six adsorption/desorptions, eight elementary surface reactions on both terrace and step sites, and five surface diffusion steps between terrace and step 
sites. The DFT data were converted into thermodynamic and kinetic data using the Python Multiscale Thermodynamic Toolbox (pMuTT).(53) Gas species data was obtained from NIST,(54) while reaction enthalpies and associated reaction barriers at each strain were used to determine BEP relationships for each surface reaction.

Haber-Bosch operating conditions $\left(400{ }^{\circ} \mathrm{C}, 200\right.$ atm, $4 \mathrm{sec}$ ) with a stoichiometric feed of $\mathrm{H}_{2}: \mathrm{N}_{2}$ of 3:1 were compared to milder conditions at both static strain (steady state operation at a single strain value) and dynamic strain (strain oscillated symmetrically with a square wave between $+4 \%$ and $-4 \%$ ) at multiple frequencies in both a flow (continuous stirred-tank reactor) and batch reactors (static and dynamic equilibrium studies). The simulations were carried out in a custom-made Matlab code. This model accounts for multiple catalyst active sites, surface coverage effects, surface reactions on both step and terrace sites, and surface diffusion between catalyst sites. The results were analyzed using reaction path analysis (the ReNView visualization tool(55)) to compute the reaction flux and the partial equilibrium index
(PEI),(56) i.e., the distance from equilibrium, for all reactions and via sensitivity analysis to identify the rate-determining steps.

\subsection{Results and Discussion.}

The straining of $\mathrm{Ru}( \pm 4 \%)$ in two dimensions across the surface, depicted in Figure 1B, alters the physical and electronic state of the catalyst. Interatomic spacing across the surface was adjusted from its relaxed state of $2.74 \AA$ down to $2.63 \AA$ under compressive strain and $2.84 \AA$ under tension. Step site interatomic spacing exhibits anisotropic behavior expanding from the relaxed condition of $2.74 \AA$ to $2.63 \AA$ and $2.84 \AA$ under compression and tension, respectively, along the step edge while expanding from $2.64 \AA$ to $2.63 \AA$ and $2.66 \AA$ under compression and tension perpendicular to the edge. Perpendicular to the step edge atoms are not restrained by nearest-neighbor atoms and, therefore, relax closer to an unstrained position. The shift in relative atomic position alters the density of states (Figure 1C) and shifts the d-band center by about $\pm 0.23 \mathrm{eV}$ over the full range of strain (Figure S30 and SI sections: 'Density of
A
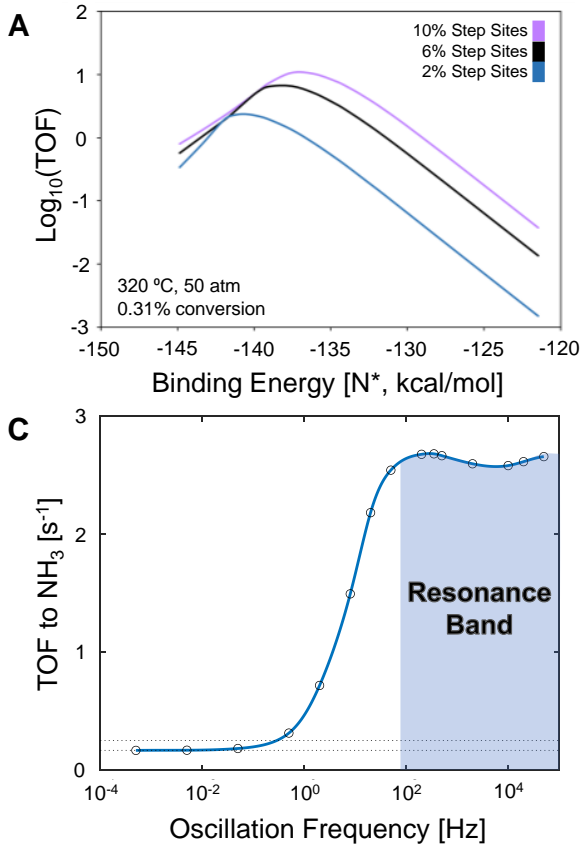

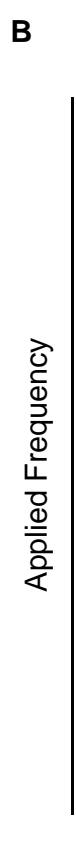

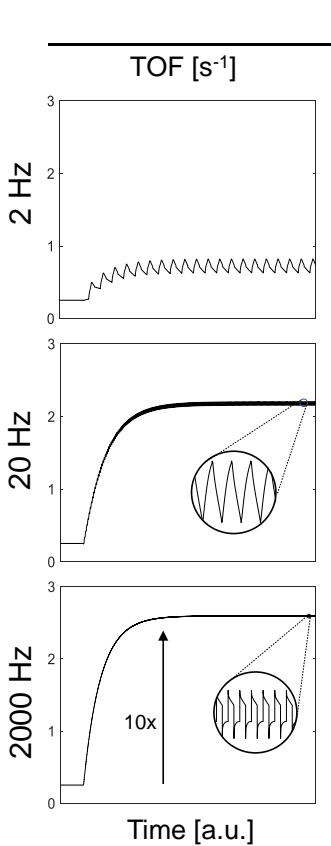

Time [a.u.]
2\% Step Sites

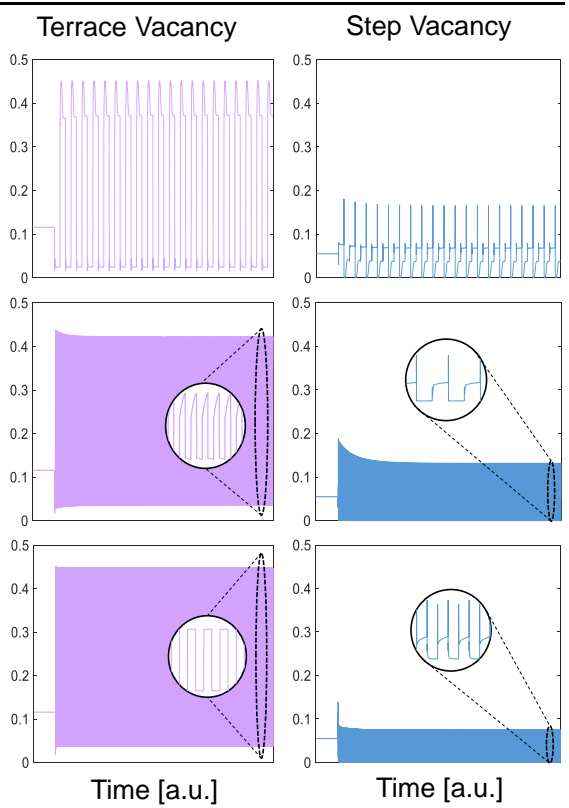

Figure 2. Dynamic oscillation of Ru strain for catalytic ammonia synthesis compared to a static volcano. A. Sabatier static strain volcano catalytic turnover frequency (TOF) at $0.31 \%$ conversion of $\mathrm{N}_{2}$ on $\mathrm{Ru}$ nanoparticles at step site densities indicated; full details in the Supplementary Information, 'Terrace and Step Site Densities'. B. Instantaneous catalytic turnover frequency and terrace and step site vacancy at various frequencies. $\mathbf{C}$. TOF under applied dynamic strain frequency $(0.31 \%$ conversion at steady state; $2 \%$ step sites $)$. All calculations are done at 320 ${ }^{\circ} \mathrm{C}$ and $50 \mathrm{~atm}$. 
States (DOS) and d-Band Center', and 'DFT Calculations').

These electronic variations manifest in variations of the binding energy $(\Delta \mathrm{G})$ of the surface species involved in ammonia synthesis. As depicted in Figure 1D, the binding energy of each adsorbate $\mathrm{NH}_{\mathrm{x}}$ on a terrace site at standard temperature and pressure (taken here as a negative quantity) increases with the degree of hydrogenation and with the $\mathrm{N}(\mathrm{T})$ binding energy on a clean (low coverage) $\mathrm{Ru}$ terrace site. In sharp contrast to $\mathrm{N}(\mathrm{T})$, the binding energy of $\mathrm{NH}_{\mathrm{x}}$ surface species varies less over the full range of strain. While $\mathrm{NH}_{\mathrm{x}}$ species binding energies on step sites are similar to terrace site, the anisotropic behavior of the step edge under strain results in a correspondingly lower strain impact on the binding energies. The relative change is defined as $\gamma<1$, where $\gamma$ is the ratio of binding energies of surface adsorbates $\left(\gamma=\Delta \Delta \mathrm{H}_{\mathrm{NH}} / \Delta \Delta \mathrm{H}_{\mathrm{N}}\right)$. The change upon straining is larger for adsorbates that interact closer and stronger with the surface.

The binding energy also varies with the binding site (terrace vs. step sites) and surface coverage. As depicted in Figure 1E, nanoparticles of ruthenium exhibit step sites (orange) which promote $\mathrm{N}_{2}(\mathrm{~T}$ ) dissociation to $\mathrm{N}(\mathrm{T})$; the fraction of step sites varies from $2 \%$ to $8 \%$ for nanoparticles of diameters between 8.0 and $2.6 \mathrm{~nm}$ (Figure S26B). Binding energies for adsorbates are impacted by nearest neighbor adsorbates. Mean-field models reflect the

A

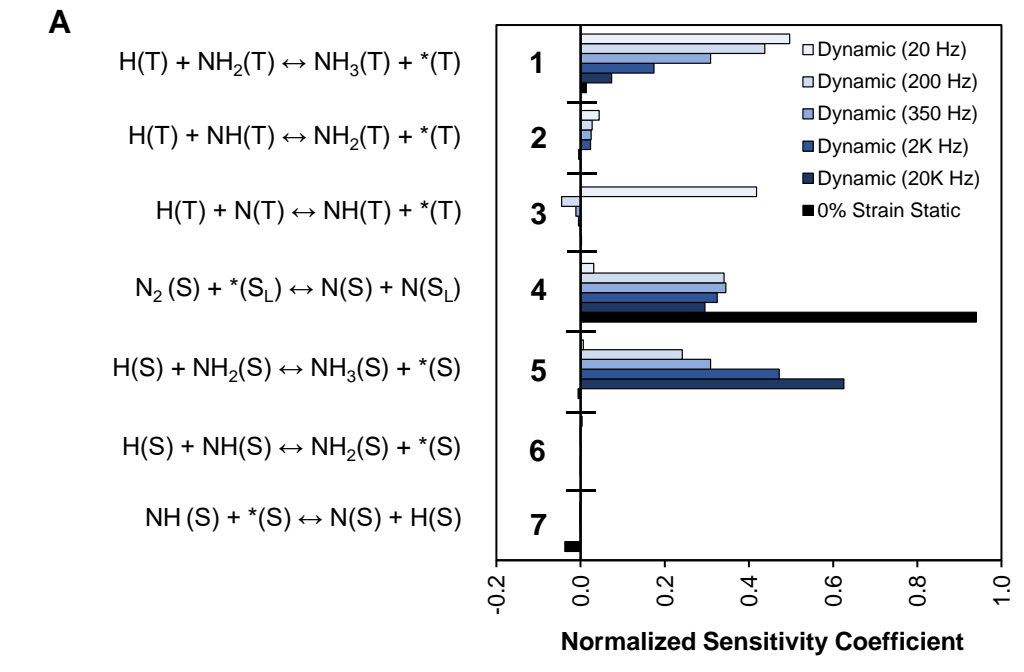

effect of adsorbate-adsorbate interactions by including an average interaction between adsorbates versus the fractional monolayer coverage of a species. When binding energies are high, as those of $\mathrm{N}$ and $\mathrm{NH}$, the lateral interaction between adsorbates is also high (Table S1). As surface coverages vary with changing strain levels (Figure S16), the impact on all adsorbates is significant (Figure S17), especially when dynamic square wave oscillations result in surface vacancies changing as much as $40 \%$ (Figure $2 \mathrm{~B}$ and SI section: 'Dynamic Simulations for Additional Results').

The kinetics of ammonia synthesis were evaluated within a multi-site microkinetic model using the calculated surface energies of ammoniaforming species under varying conditions of strain, pressure, and temperature. Reactions of $\mathrm{N}_{2}$ dissociation and $\mathrm{N}^{*}$ hydrogenation occur on both step and terrace sites with molecules diffusing between sites. Reactions occur within a perfectly mixed continuous flow tank reactor, whose space velocity was varied to reach a steady-state conversion of $\mathrm{N}_{2}$ of $0.31 \%$ (differential conditions). Full details are provided in the Supporting Information and Methods Sections (SI sections: 'Reaction Mechanism' and 'Thermodynamics, Kinetics, and Coverage Effects').

The kinetics of the simulated ammonia synthesis reaction on static $\mathrm{Ru}$ exhibits strain volcanoes as depicted in Figure $2 \mathrm{~A}$ at $320^{\circ} \mathrm{C}$ and

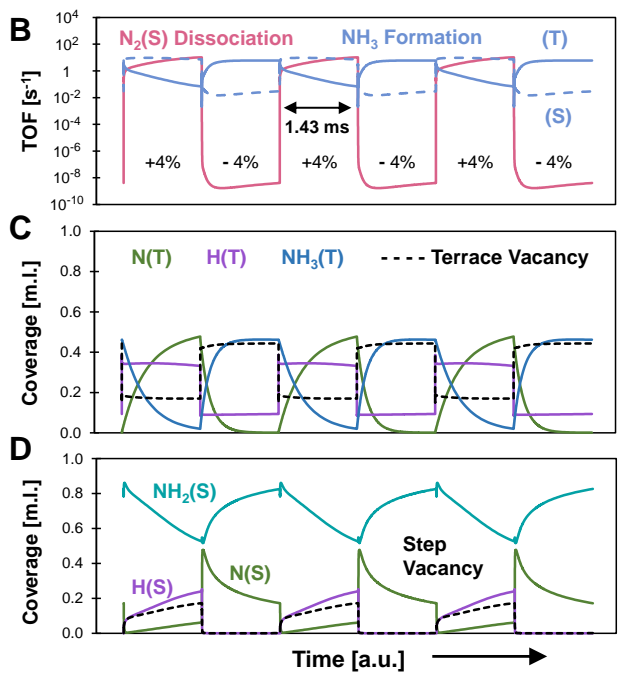

Figure 3. Rate limitations of ammonia synthesis on dynamically strained Ru. (A) Sensitivity analysis for the rate-relevant catalytic steps in ammonia synthesis for varying applied frequencies. $\mathrm{S}$ and $\mathrm{S}_{\mathrm{L}}$ indicate the upper and lower step sites and * a vacancy. (B) Dynamic flux, (C) terrace (T) surface coverage, and (D) upper step (S) surface coverage of ammonia synthesis at $350 \mathrm{~Hz}$. All panels: $320^{\circ} \mathrm{C}, 50 \mathrm{~atm}, 2 \%$ step sites. 
A

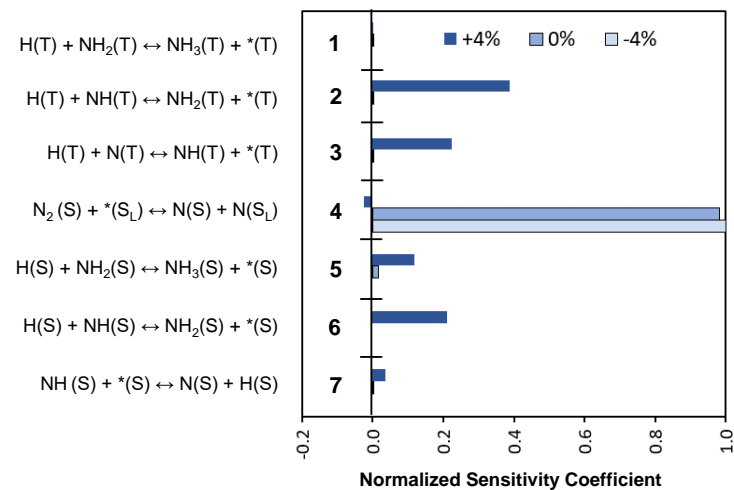

C

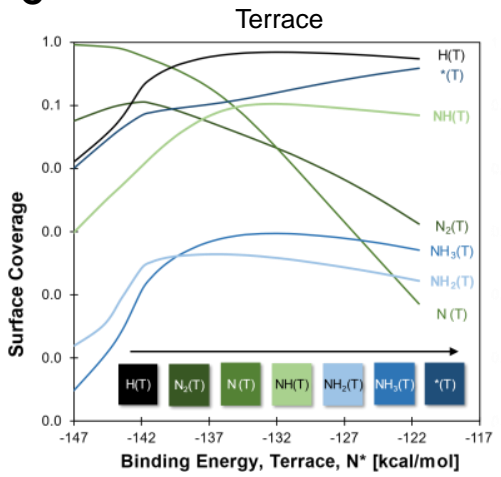

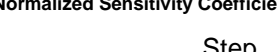

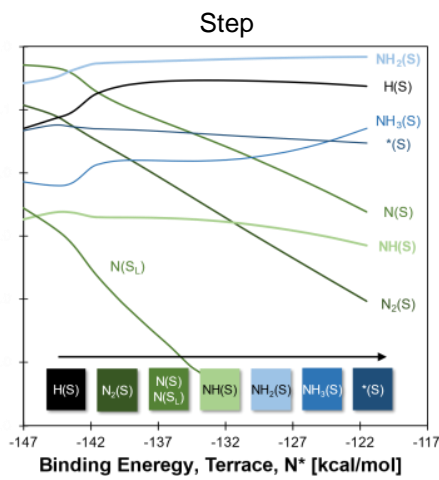

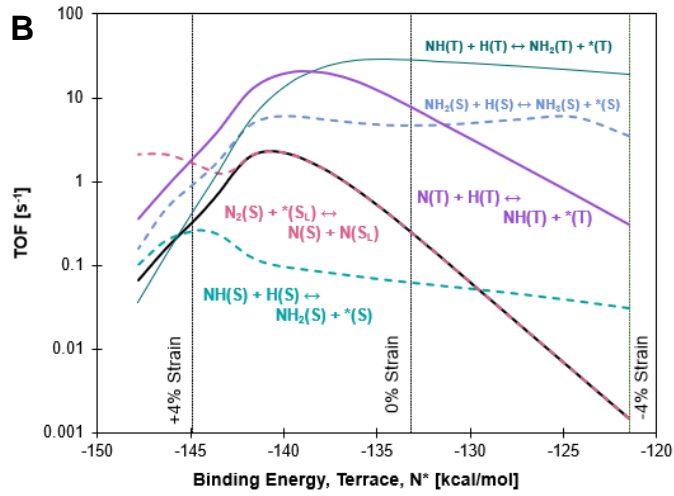

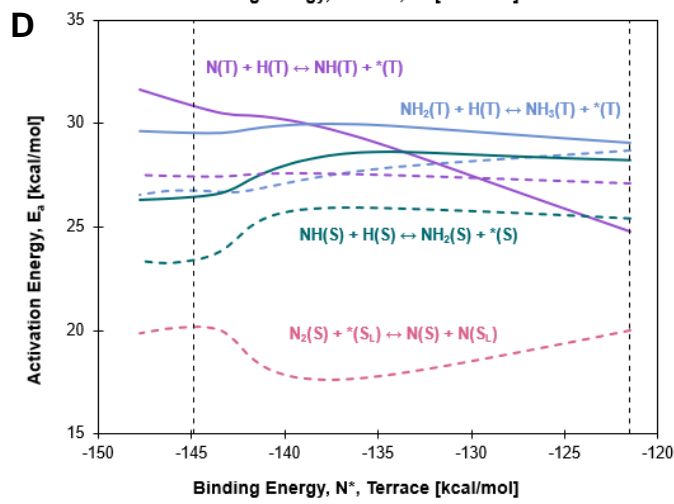

Figure 4. Rate limitations of ammonia synthesis on statically strained Ru. A. Sensitivity of ammonia synthesis at three strains indicated. B. Static strain-affected TOF for potentially rate-determining reactions comprising the composite volcano curve on upper step (dashed) and terrace (line) sites. C. Terrace and upper step site coverage (fraction of monolayer) as impacted by static strain. D. Static strain impact on reaction barriers on upper step (dashed) and terrace (line) sites. Conditions of $320^{\circ} \mathrm{C}$ and 50 atm with $2 \%$ Ru step sites. $\mathrm{S}$ and $\mathrm{S}_{\mathrm{L}}$ indicate the upper and lower step sites and * a vacancy.

$50 \mathrm{~atm}$. Unique from the volcanoes for various metal surfaces in Figure 1A, the strain volcano peaks shift with the density of steps to $140.7,138.2$, and $137.1 \mathrm{kcal} / \mathrm{mol}$ (on the $\mathrm{N}(\mathrm{T})$ terrace binding energy scale) for 2, 6, and $10 \%$ step sites, respectively. Larger nanoparticles (fewer step sites) shift the strain volcano peak toward conditions of higher tensile strain (a lower N(T) binding energy).

Dynamic simulations using a symmetric square wave oscillatory strain $( \pm 4 \%)$ between both sides of the strain volcano (Figure 2B for a $\mathrm{Ru}$ surface comprised of $2 \%$ step sites and SI sections: 'Terrace and Step Site Densities' and 'Matlab Kinetic Model') exhibit complex frequency response in turnover frequency (TOF) and terrace and step site vacancies. Upon transition from static to dynamic conditions, the catalytic rate increases before achieving a periodic solution (i.e., a stable limit cycle). Under these conditions, the surface coverages are changing substantially for each applied oscillation, with terrace and step site vacancy changing as much as $40 \%$ and $10 \%$, respectively, with even higher surface coverage changes for individual adsorbates $(\mathrm{N}(\mathrm{T})$ coverage changes as much as $90 \%$ ). At $2 \mathrm{kHz}$, the catalytic rate increases one order of magnitude. Rate acceleration begins above about $1 \mathrm{~Hz}$ and achieves resonance at 100 to $10^{5} \mathrm{~Hz}$ and beyond (Figure 2C).

Rate enhancement via dynamic strain is interpreted via sensitivity analysis of elementary reaction steps. The impact of seven elementary reactions on Ru step (S) and terrace (T) sites, shown in Figure 3A, on the overall rate of ammonia synthesis was evaluated by modifying the preexponential factors (by $\pm 2 \%$ ). Under static conditions (zero strain), the rate-determining step is the scission of adsorbed $\mathrm{N}_{2}(\mathrm{~S})$ on the Ru steps (step 4). Under slow oscillation $(20 \mathrm{~Hz})$, the rate control shifts to $\mathrm{NH}_{2}(\mathrm{~T})$ and $\mathrm{N}(\mathrm{T})$ hydrogenation (steps 1 

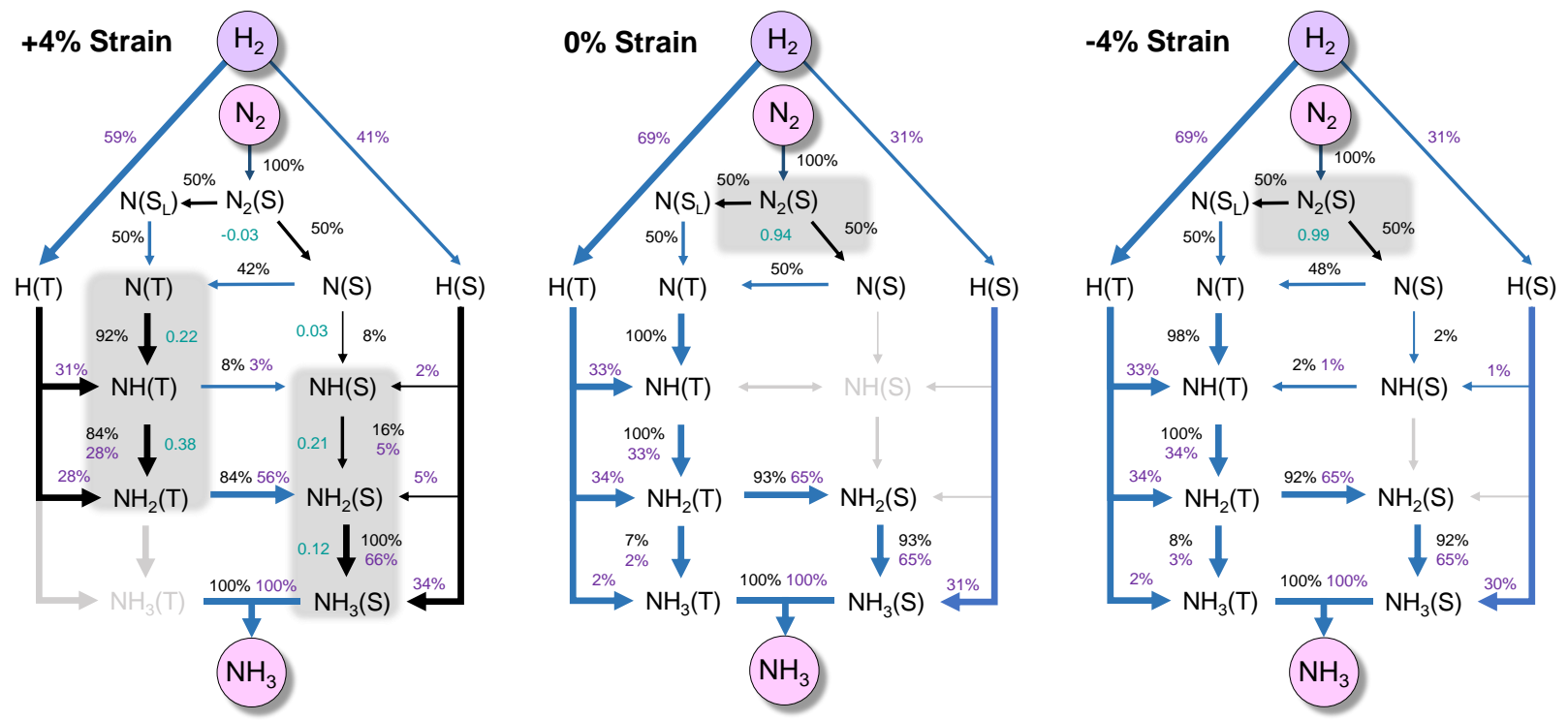

Figure 5. Reaction paths of catalytic ammonia synthesis. $+4 \%$ (A), $0 \%$ (B) and $-4 \%$ (C) strain. Numbers represent the percent of molar flux along that arrow relative to $\mathrm{N}_{2}$ gas (black text) or $\mathrm{H}_{2}$ gas (purple text) adsorbing on the surface. The normalized sensitivity coefficient of each path is indicated in teal text. Grey species and lines are reactions and species in the mechanism with de minimis reaction flux for a given static strain. (T) - Terrace, (S) Upper Step, $\left(\mathrm{S}_{\mathrm{L}}\right)$ - Lower Step

and 3). Higher frequencies at resonance conditions $(>100 \mathrm{~Hz})$ shift the rate control to $\mathrm{N}_{2}(\mathrm{~S})$ dissociation (step 4) and $\mathrm{NH}_{2}(\mathrm{~T})$ and $\mathrm{NH}_{2}(\mathrm{~S})$ hydrogenation (steps 1 and 5). This indicates that rate enhancement is associated with a shift in the rate-determining step. The shift in $\mathrm{NH}_{2}$ hydrogenation from steps to terraces also demonstrates how dynamics couples to individual reaction steps. The hydrogenation reaction, under static strain, is three orders-of-magnitude faster on steps than on terraces. At $20 \mathrm{~Hz}$, the oscillations couple with the slower terrace reaction increasing the ammonia TOF with little impact on the step site reaction, while the higher frequency oscillations $(20 \mathrm{kHz})$ couple with the faster step site reaction increasing the TOF with negligible influence on the terrace site reaction.

The transition in rate limitation also appears in the surface coverages at $350 \mathrm{~Hz}$ (Figure 3B-3D). At this frequency, the catalytic system proceeds at each extreme strain condition $( \pm 4 \%)$ for 1.43 milliseconds, allowing for the steps and terraces to almost transition to the new strain condition. However, the surface species never achieve steady state before reverting to the next strain condition, consistent with its overall catalytic TOF within the resonance range $\left(10^{2}\right.$ to $\left.10^{5+} \mathrm{Hz}\right)$. At $+4 \%$ strain, $\mathrm{N}_{2}(\mathrm{~S})$ readily dissociates on the steps accumulating nitrogen on the step and adjacent terrace sites, $\mathrm{N}(\mathrm{S})$ and N(T). Simultaneously, hydrogen, dissociates on both Ru sites, as $\mathrm{H}(\mathrm{T})$ and $\mathrm{H}(\mathrm{S})$, proportional to the hydrogenation fluxes. When reverting to negative $(-4 \%)$ strain, the nitrogen and hydrogen atoms are depleted, and $\mathrm{NH}_{3}(\mathrm{~T})$ and $\mathrm{NH}_{2}(\mathrm{~S})$ accumulate on the surface. Oscillating between these two conditions produces ammonia from both step and terrace surface sites, unlike the common static finding of ammonia chemistry proceeding only on steps.

Further interpretation of dynamic rate enhancement requires analysis under static strain (Figure 4). Sensitivity analysis identifies $\mathrm{N}_{2}$ dissociation on steps as rate-limiting at $-4 \%$ and $0 \%$ strain (Figure 4A). At $+4 \%$ strain, the overall TOF is higher (Figure 2A), and reactions 2, 3, 5, and 6 are all important; the $\mathrm{N}_{2}$ dissociation is no longer rate-limiting. It follows that the higher stability of $\mathrm{N}_{2}(\mathrm{~S})$ associated with the higher adsorbate binding energy at $+4 \%$ strain promotes the $\mathrm{N}_{2}$ dissociation, thereby shifting the rate-determining step further down the catalytic cycle. Individual elementary reaction rates (Figure 4B) provide insight into the reaction fluxes contributing to the overall static strain (composite volcano curve). The adsorption, desorption, and surface diffusions are fast relative to the overall catalytic reaction (Figures S33A-B). 

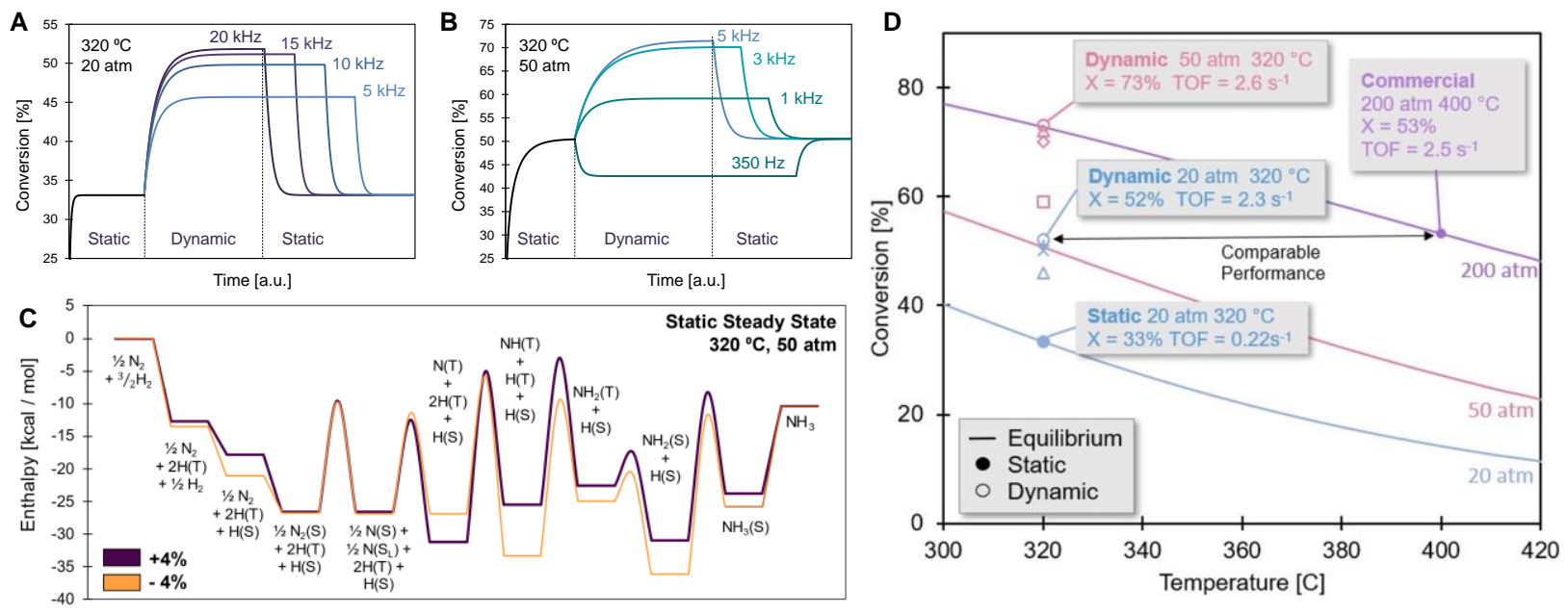

Figure 6. Catalyst dynamic operation away from equilibrium. A. Surface dynamics ( $\pm 4 \%$ square wave strain) initiated at time zero at various frequencies promotes ammonia synthesis above the equilibrium conversion of $33 \%$ at $320^{\circ} \mathrm{C}$ and $20 \mathrm{~atm}$ to a periodic solution as high as $52 \%$ at $20 \mathrm{kHz}$. B. Same as A but at $50 \mathrm{~atm}$, the conversion is as high as $73 \%$ at $5 \mathrm{kHz}$. C. The static reaction enthalpy diagram of ammonia synthesis on Ru at $\pm 4 \%$ strain with steadystate coverage effects included. D. Comparison of static $(\bullet)$ and dynamic $(\circ 10 \mathrm{kHz} \& 20 \mathrm{kHz}, \diamond 3.0 \mathrm{kHz}, \square 1.0 \mathrm{kHz}$, $\Delta 5.0 \mathrm{kHz},+15 \mathrm{kHz}, \times 10 \mathrm{kHz}$; blue points for $20 \mathrm{~atm}$ and red points for $50 \mathrm{~atm}$ ) ammonia synthesis with equilibrium (lines) vs. temperature at indicated pressures. $(\mathrm{T})$ - Terrace, $(\mathrm{S})$ - Upper Step, $\left(\mathrm{S}_{\mathrm{L}}\right)$ - Lower Step

The $\mathrm{N}_{2}$ dissociation on steps fully defines the volcano between a strain of $3.25 \%$ to $-4 \%$ (Figure $3 \mathrm{~B}$ ), consistent with the sensitivity analysis (Figure $4 \mathrm{~A})$. For $+4 \%$ strain, the combination of reactions $2,3,5$, and 6 defines the left side of the strain volcano. Thus, the strain volcano is associated with a shift in rate control between $\mathrm{N}_{2}(\mathrm{~S})$ dissociation and $\mathrm{NH}_{\mathrm{x}}$ hydrogenation. The oscillatory operation discussed above overcomes the $\mathrm{N}_{2}(\mathrm{~S})$ dissociation limitation, seen under most conditions of the static strain, to enhance the reaction rate.

The shift between $\mathrm{N}_{2}$ dissociation as the ratedetermining step to the hydrogenation is accompanied by an $\mathrm{N}(\mathrm{S})$ coverage increase and $\mathrm{H}(\mathrm{S})$ decrease (Figure 4C) when transitioning from negative to positive strain. At large positive strain (most negative binding energy), $\mathrm{N}(\mathrm{S})$ and $\mathrm{N}(\mathrm{T})$ are the dominant surface species, with $\mathrm{H}(\mathrm{S})$ no longer blocking the active sites. These shifts in surface coverage combined with the moderate alterations in activation energy (Figure 4D) change the ratedetermining steps (summarized in Figure 5) and enable the dynamic catalytic rate improvement.

The dynamic strain results in a periodic conversion differing from equilibrium, consistent with other dynamic ratcheted systems. $(21,57)$ In Figure 6A, the ammonia synthesis reaction was simulated in a batch reactor at $320{ }^{\circ} \mathrm{C}$ and $20 \mathrm{~atm}$ starting with a stoichiometric mixture of $\mathrm{H}_{2}$ and $\mathrm{N}_{2}$ gases. Initial static catalysis proceeds to $33 \%$ conversion at equilibrium, after which dynamic strain $( \pm 4 \%)$ at varying frequency $(5,10,15$, and $20 \mathrm{kHz}$ ) was imposed. Dynamic operation increases the conversion to $45.7,49.8,51.2$, and $51.8 \%$, after which the catalyst returns to static operation and the chemistry proceeds to equilibrium. At higher pressure of $50 \mathrm{~atm}$ in Figure 6B, variation of strain $( \pm 4 \%)$ at lower frequencies $(350 \mathrm{~Hz}, 1,3$, and 5 $\mathrm{kHz}$ ) profoundly increases the conversion above equilibrium.

Interpreting the shift in conversion of ammonia synthesis away from equilibrium under dynamic strain is complicated by the number of sequential elementary steps in the overall mechanism. As depicted in Figure 6B, strain oscillation can promote ammonia synthesis or decomposition (forward or reverse chemistry), depending on the applied frequency. Prior simulations of model catalytic systems (A-to-B) identified simple criteria for assessing the directionality of dynamic mechanisms (i.e., the delta parameter of a single elementary reaction).(20) However, ammonia synthesis exhibits a broad range of dynamic behaviors; as depicted in Figure 6C, the reaction enthalpy diagrams of both positive and negative $\pm 4 \%$ strain depict intermediate surface species 
energies across common transition states that vary uniquely (i.e., positive and negative gamma). This includes reactions like $\mathrm{N}^{*}$ hydrogenation, for which negative $\gamma$ dynamic behavior is observed; the surface energies of $\mathrm{N}(\mathrm{T})$ and $\mathrm{NH}(\mathrm{T})$ change in opposite directions under variable strain. No overarching resonance theory yet exists to explain the overall directionality of complex reaction mechanisms.

Our proposed dynamic strain model demonstrates that catalytic resonance theory can be applied to complex reaction mechanisms and achieve rate and conversion enhancement. While the initial simulations of catalytic resonance only accounted for overall reactions determined by single elementary reactions, $(19,20)$ the ammonia synthesis mechanism accounts for multiple pathways on two sites exchanging molecules via surface diffusion and lateral interactions of adsorbates, exhibiting linear scaling relationships in binding energy and transition state energy. Despite the inherent complexity of ammonia surface chemistry, the general predictions of catalytic resonance are retained.

Implementation of dynamic strain will require optimization of the applied dynamic strain waveform beyond the single frequency square wave oscillations employed here. The timescales in the ammonia synthesis steps (Figure 6C) indicate that more complex waveforms (amplitudes and frequencies) could improve the reaction. Moreover, comparing the model with experiment will require including the transverse and longitudinal surface waves arising from different methods of surface perturbation(21) that introduce different types of local surface strain (one- or two-dimensional, varying local curvature). These improvements in applied dynamic strain waveform can further enhance ammonia synthesis beyond the order of magnitude rate increase and pressure decrease predicted here.

Acknowledgements. We acknowledge the financial support of the Catalysis Center for Energy Innovation, a U.S. Department of Energy - Energy Frontier Research Center under Grant DESC0001004 that has been the intellectual driver for this work. The code for this work was supported by the Department of Energy's Office of Energy Efficient and Renewable Energy's Advanced
Manufacturing Office under Award Number DEEE0007888-9.5. The Delaware Energy Institute gratefully acknowledges the support and partnership of the State of Delaware toward the RAPID projects. We are also grateful to Drs. David Flannigan, Omar Abdelrahman, and Stavros Caratzoulas for helpful discussions and to Sophia Kurdziel and Seungyeon Lee for their assistance with the validation of transition states and density of states data, respectively.

Keywords. Ammonia, Ruthenium, Strain, Dynamics, Resonance.

Supporting Information. Additional information including setup of the microreactor is located in the online supporting information.

\section{References}

1. N. M. Haegel, H. Atwater, T. Barnes, C. Breyer, A. Burrell, Y. M. Chiang, S. De Wolf, B. Dimmler, D. Feldman, S. Glunz, J. C. Goldschmidt, D. Hochschild, R. Inzunza, I. Kaizuka, B. Kroposki, S. Kurtz, S. Leu, R. Margolis, K. Matsubara, A. Metz, W. K. Metzger, M. Morjaria, S. Niki, S. Nowak, I. M. Peters, S. Philipps, T. Reindl, A. Richter, D. Rose, K. Sakurai, R. Schlatmann, M. Shikano, W. Sinke, R. Sinton, B. J. Stanbery, M. Topic, W. Tumas, Y. Ueda, J. Van De Lagemaat, P. Verlinden, M. Vetter, E. Warren, M. Werner, M. Yamaguchi, A. W. Bett, Terawatt-scale photovoltaics: Transform global energy Improving costs and scale reflect looming opportunities. Science. 364, 836-838 (2019).

2. R. Service, Ammonia - a renewable fuel made from sun, air, and water - could power the globe without carbon. Science. 361, 120-123 (2018).

3. Alexander H. Tullo, Is ammonia the fuel of the future? Chem. Eng. News. 99, 20-22 (2021).

4. M. J. Palys, P. Daoutidis, Using hydrogen and ammonia for renewable energy storage: A geographically comprehensive techno-economic study. Comput. Chem. Eng. 136 (2020).

5. M. J. Palys, A. Allman, P. Daoutidis, 
Exploring the Benefits of Modular Renewable-Powered Ammonia Production: A Supply Chain Optimization Study. Ind. Eng. Chem. Res. 58, 5898-5908 (2019).

6. M. J. Palys, A. McCormick, E. L. Cussler, P. Daoutidis, Modeling and optimal design of absorbent enhanced ammonia synthesis. Processes. 6 (2018).

7. A. Vojvodic, J. K. Nørskov, New design paradigm for heterogeneous catalysts. Natl. Sci. Rev. 2, 140-143 (2015).

8. A. Logadottir, T. . H. Rod, J. . K. Nørskov, B. Hammer, S. Dahl, C. J. . J. H. Jacobsen, The Brønsted-Evans-Polanyi Relation and the Volcano Plot for Ammonia Synthesis over Transition Metal Catalysts. J. Catal. 197, 229-231 (2001).

9. K. Honkala, A. Hellman, I. N. Remediakis, A. Logadottir, A. Carlsson, S. Dahl, C. H. Christensen, J. K. Nørskov, Ammonia synthesis from first-principles calculations. Science. 4963, 2003-2006 (2005).

10. S. Dahl, J. Sehested, C. J. H. Jacobsen, E. Törnqvist, I. Chorkendorff, Surface science based microkinetic analysis of ammonia synthesis over ruthenium catalysts. $J$. Catal. 192, 391-399 (2000).

11. Á. Logadóttir, J. K. Nørskov, Ammonia synthesis over a $\mathrm{Ru}(0001)$ surface studied by density functional calculations. J. Catal. 220, 273-279 (2003).

12. A. J. Medford, A. Vojvodic, J. S. Hummelshøj, J. Voss, F. Abild-Pedersen, F. Studt, T. Bligaard, A. Nilsson, J. K. Nørskov, From the Sabatier principle to a predictive theory of transition-metal heterogeneous catalysis. J. Catal. 328, 3642 (2015).

13. A. J. Martín, T. Shinagawa, J. PérezRamírez, Electrocatalytic Reduction of Nitrogen: From Haber-Bosch to Ammonia Artificial Leaf. Chem. 5, 263-283 (2019).

14. J. G. Chen, R. M. Crooks, L. C. Seefeldt, K. L. Bren, R. Morris Bullock, M. Y. Darensbourg, P. L. Holland, B. Hoffman, M. J. Janik, A. K. Jones, M. G. Kanatzidis, P. King, K. M. Lancaster, S. V. Lymar, P. Pfromm, W. F. Schneider, R. R. Schrock, Beyond fossil fuel-driven nitrogen transformations. Science. 360 (2018).

15. W. Guo, D. G. Vlachos, Effect of local metal microstructure on adsorption on bimetallic surfaces: Atomic nitrogen on NiPt(111). J. Chem. Phys. 138, 2-4 (2013).

16. W. Guo, D. G. Vlachos, Patched bimetallic surfaces are active catalysts for ammonia decomposition. Nat. Commun. 6, 1-7 (2015).

17. W. Guo, M. Stamatakis, D. G. Vlachos, Design principles of heteroepitaxial bimetallic catalysts. ACS Catal. 3, 22482255 (2013).

18. M. Núñez, D. G. Vlachos, J. L. Lansford, D. G. Vlachos, Optimization of the facet structure of transition-metal catalysts applied to the oxygen reduction reaction. Nat. Chem. 11, 449-456 (2019).

19. M. A. Ardagh, O. A. Abdelrahman, P. J. Dauenhauer, Principles of Dynamic Heterogeneous Catalysis: Surface Resonance and Turnover Frequency Response. ACS Catal. 9, 6929-6937 (2019).

20. M. A. Ardagh, T. Birol, Q. Zhang, O. A. Abdelrahman, P. J. Dauenhauer, Catalytic resonance theory: SuperVolcanoes, catalytic molecular pumps, and oscillatory steady state. Catal. Sci. Technol. 9, 50585076 (2019).

21. M. Shetty, A. Walton, S. R. Gathmann, M. A. Ardagh, J. Gopeesingh, J. Resasco, T. Birol, Q. Zhang, M. Tsapatsis, D. G. Vlachos, P. Christopher, C. D. Frisbie, O. A. Abdelrahman, P. J. Dauenhauer, The Catalytic Mechanics of Dynamic Surfaces: Stimulating Methods for Promoting Catalytic Resonance. ACS Catal. 10, 12666-12695 (2020).

22. P. J. Dauenhauer, M. A. Ardagh, M. Shetty, A. Kuznetsov, Q. Zhang, P. Christopher, D. G. Vlachos, O. A. Abdelrahman, Catalytic resonance theory: Parallel reaction pathway control. Chem. Sci. 11, 3501-3510 (2020).

23. J. Gopeesingh, M. A. Ardagh, M. Shetty, S. T. Burke, P. J. Dauenhauer, O. A. Abdelrahman, Resonance-Promoted Formic Acid Oxidation via Dynamic Electrocatalytic Modulation. ACS Catal. 10, 9932-9942 (2020).

24. J. Qi, J. Resasco, H. Robatjazi, I. B. Alvarez, O. Abdelrahman, P. Dauenhauer, 


\section{ChemRxiv}

P. Christopher, Dynamic Control of Elementary Step Energetics via Pulsed Illumination Enhances Photocatalysis on Metal Nanoparticles. ACS Energy Lett. 5, 3518-3525 (2020).

25. M. Mavrikakis, B. Hammer, J. K. Nørskov, Effect of Strain on the Reactivity of Metal Surfaces. Phys. Rev. Lett. 81, 2819-2822 (1998).

26. A. Khorshidi, J. Violet, J. Hashemi, A. A. Peterson, How strain can break the scaling relations of catalysis. Nat. Catal. 1, 263268 (2018).

27. J. R. Kitchin, Correlations in coveragedependent atomic adsorption energies on Pd(111). Phys. Rev. B - Condens. Matter Mater. Phys. 79, 1-6 (2009).

28. J. Hwang, Z. Feng, N. Charles, X. R. Wang, D. Lee, K. A. Stoerzinger, S. Muy, R. R. Rao, D. Lee, R. Jacobs, D. Morgan, Y. Shao-Horn, Tuning perovskite oxides by strain: Electronic structure, properties, and functions in (electro)catalysis and ferroelectricity. Mater. Today. 31, 100-118 (2019).

29. Y. Xu, M. Mavrikakis, Adsorption and dissociation of $\mathrm{O} 2$ on gold surfaces: Effect of steps and strain. J. Phys. Chem. B. 107, 9298-9307 (2003).

30. F. Liu, C. Wu, G. Yang, S. Yang, CO Oxidation over Strained Pt(100) Surface: A DFT Study. J. Phys. Chem. C. 119, 1550015505 (2015).

31. Y. Xu, M. Mavrikakis, Adsorption and dissociation of $\mathrm{O} 2$ on $\mathrm{Cu}\left(\begin{array}{lll}1 & 1 & 1\end{array}\right)$ :

Thermochemistry, reaction barrier and the effect of strain. Surf. Sci. 494, 131-144 (2001).

32. J. H. Larsen, I. Chorkendorff, Increased dissociation probability of $\mathrm{CH} 4$ on $\mathrm{Co} / \mathrm{Cu}(111)$. Surf. Sci. 405, 62-73 (1998).

33. V. A. Sethuraman, D. Vairavapandian, M. C. Lafouresse, T. Adit Maark, N. Karan, S. Sun, U. Bertocci, A. A. Peterson, G. R. Stafford, P. R. Guduru, Role of Elastic Strain on Electrocatalysis of Oxygen Reduction Reaction on Pt. J. Phys. Chem. C. 119, 19042-19052 (2015).

34. A. Schlapka, M. Lischka, A. Groß, U. Käsberger, P. Jakob, Surface Strain versus Substrate Interaction in Heteroepitaxial
Metal Layers: Pt on Ru(0001). Phys. Rev. Lett. 91, 016101 (2003).

35. J. R. Kitchin, J. K. Nørskov, M. A. Barteau, J. G. Chen, Modification of the surface electronic and chemical properties of $\mathrm{Pt}(111)$ by subsurface $3 \mathrm{~d}$ transition metals. J. Chem. Phys. 120, 10240-10246 (2004).

36. J. X. Wang, H. Inada, L. Wu, Y. Zhu, Y. M. Choi, P. Liu, W. P. Zhou, R. R. Adzic, Oxygen reduction on well-defined coreshell nanocatalysts: Particle size, facet, and Pt shell thickness effects. J. Am. Chem. Soc. 131, 17299-17302 (2009).

37. H. Wang, S. Xu, C. Tsai, Y. Li, C. Liu, J. Zhao, Y. Liu, H. Yuan, F. Abild-Pedersen, F. B. Prinz, J. K. Nørskov, Y. Cui, Direct and continuous strain control of catalysts with tunable battery electrode materials. Science. 354, 1031-1036 (2016).

38. K. Yan, S. K. Kim, A. Khorshidi, P. R. Guduru, A. A. Peterson, High Elastic Strain Directly Tunes the Hydrogen Evolution Reaction on Tungsten Carbide. J. Phys. Chem. C. 121, 6177-6183 (2017).

39. Y. Inoue, Acoustic enhancement of surface reactions. MRS Bull. 44, 361-371 (2019).

40. H. Nishiyama, Y. Inoue, PEEM study of work function changes in $\mathrm{Cu}, \mathrm{Au}$ and $\mathrm{Pd}$ metal surfaces with surface acoustic wave propagation. Surf. Sci. 600, 2644-2649 (2006).

41. Y. Inoue, M. Matsukawa, K. Sato, Effect of Surface Acoustic Wave Generated on Ferroelectric Support upon Catalysis. J. Am. Chem. Soc. 111, 8965-8966 (1989).

42. H. Nishiyama, N. Saito, M. Shima, Y. Watanabe, Y. Inoue, Effects of acoustic waves on activation of thin film $\mathrm{Pd}$ and $\mathrm{Ni}$ catalysts for ethanol and $\mathrm{CO}$ oxidation. Faraday Discuss. 107, 425-434 (1997).

43. N. F. Morozov, L. S. Shikhobalov, Effect of impact loading duration on yield strength. Dokl. Phys. 53, 529-532 (2008).

44. L. Zhang, E. K. H. Salje, X. Ding, J. Sun, Strain rate dependence of twinning avalanches at high speed impact. Appl. Phys. Lett. 104 (2014).

45. S. Dahl, A. Logadottir, R. C. Egeberg, J. H. Larsen, I. Chorkendorff, E. Törnqvist, J. K. Nørskov, Role of Steps in N2 Activation 
on $\mathrm{Ru}(0001)$. Phys. Rev. Lett. 83, 1814 1817 (1999).

46. S. Dahl, E. Tornqvist, I. Chorkendorff, Dissociative adsorption of $\mathrm{N}$ on $\mathrm{Ru}(0001)$ : A surface reaction totally dominated by steps. J. Catal. 192, 381-390 (2000).

47. G. Kresse, J. Hafner, Ab initio molecular dynamics for open-shell transition metals. Phys. Rev. B. 48, 13115-13118 (1993).

48. G. Kresse, J. Furthmüller, Efficiency of abinitio total energy calculations for metals and semiconductors using a plane-wave basis set. Comput. Mater. Sci. 6, 15-50 (1996).

49. G. Kresse, J. Furthmüller, Efficient iterative schemes for $a b$ initio total-energy calculations using a plane-wave basis set. Phys. Rev. B. 54, 11169-11186 (1996).

50. J. P. Perdew, K. Burke, M. Ernzerhof, Generalized gradient approximation made simple. Phys. Rev. Lett. 77, 3865-3868 (1996).

51. G. Henkelman, B. P. Uberuaga, H. Jónsson, Climbing image nudged elastic band method for finding saddle points and minimum energy paths. J. Chem. Phys. 113, 9901-9904 (2000).

52. H. J. Monkhorst, J. D. Pack, Special points for Brillouin-zone integrations. Phys. Rev. B. 13, 5188-5192 (1976).

53. J. Lym, G. R. Wittreich, D. G. Vlachos, A Python Multiscale Thermochemistry Toolbox (pMuTT) for thermochemical and kinetic parameter estimation. Comput. Phys. Commun. 247, 106864 (2020).

54. P. J. Linstrom, W. G. Mallard, NIST Chemistry webBook, NIST Standard Reference Database Number 69. Natl. Inst. Stand. Technol. (2013), (available at http://webbook.nist.gov/).

55. U. Gupta, D. G. Vlachos, Reaction Network Viewer (ReNView): An opensource framework for reaction path visualization of chemical reaction systems. SoftwareX. 11, 100442 (2020).

56. G. R. Wittreich, K. Alexopoulos, D. G. Vlachos, in Handbook of Materials Modeling, W. Andreoni, S. Yip, Eds. (Springer International Publishing, Cham, 2020), pp. 1377-1404.

57. P. M. Hoffman, Life's Ratchet. How
Molecular Machines Extract Order from Chaos (Basic Books, ed. 1st, 2012). 